\title{
Chromatic numbers and indices of the optimised degree six 3-modified chordal ring network topology
}

\author{
S. L. E. Chien a, R. N. Farah b, ${ }^{*}$, M. Othman ${ }^{\mathrm{c}}$ \\ ${ }^{a, b}$ Department of Mathematics, Faculty of Science and Mathematics, Universiti Pendidikan Sultan Idris, Malaysia \\ c Department of Communication Technology and Network, Faculty of Computer Science and Technology, Universiti Putra Malaysia, Malaysia \\ * Corresponding author: johnnytan125zr@gmail.com
}

\section{Article history \\ Received 21 December 2016 \\ Accepted 31 January 2017}

\section{Graphical abstract}

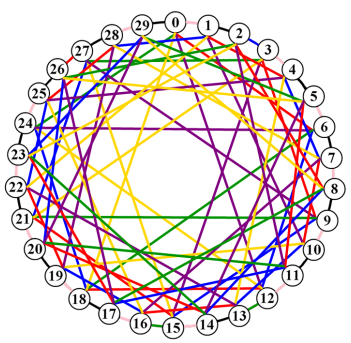

\begin{abstract}
Graph colouring is the labelling of the elements of a graph subject to certain constraints. It is divided into vertex and edge colouring. In both cases, the assignment of labels, traditionally called colours is such that two vertices or edges must not have the same colour. Graph colouring has various applications, especially in parallel computing. This paper introduces the degree six 3-modified chordal ring, $\mathrm{CHRGO}_{3}$ a parallel network interconnection topology model, and discusses its node and link colouring. Despite its asymmetry, the chromatic number of $\mathrm{CHR6o}_{3}$ cannot be generalised and must be determined specifically based on the combination of chords in each case. Cases where the chromatic index of $\mathrm{CHRGo}_{3}$ is its degree and where it is its one colour more than its degree were generalised and proven. Chromatic numbers are important in minimising the completion times of processes in parallel processing by reducing points of synchronisation, and link colouring aids processor scheduling.
\end{abstract}

Keywords: Parallel processing, modified chordal rings, graph colouring, chromatic number, chromatic index.

\section{INTRODUCTION}

One important concept in Graph Theory is graph colouring, which has gained much attention from various researchers since Francis Guthrie postulated the four colour conjecture. Graph colouring is defined as the systematic labelling of the elements of a graph subject to certain constraints [1]. It has various applications depending on the objective of the graph model considered. There are two types of graph colouring, vertex colouring and edge colouring. The objective of vertex colouring is to find a minimal number of labels for the vertices of a simple connected graph such that the same colour is not assigned to two adjacent vertices. This minimum number of labels, $k$ is called the proper colouring or chromatic number of a graph, $\chi(G)=k$. Edge colouring on the other hand, considers the minimal labelling of the graph edges in a similar manner. If there exist a minimal partition of the edge set $E(G)$ into $k$ disjoint sets such that no two edges of any edge partition are adjacent to each other, $k$ is its edge chromatic number, or chromatic index, $\chi^{\prime}(G)=k[2]$.

Chordal rings and their properties have been the focus of various authors [3], [4], [5] since their founding in 1981 [6], as they are important interconnection topology models in large parallel multiprocessor networks. A chordal ring is generally ring topology with additional chords linking non-adjacent nodes. It is based on a simple, undirected circulant graph and defined as $(N, Q)$, where $N$ is the number of nodes in the ring while $Q$ is the set of the chords of length $h_{i}$ where $h_{i} \in\left\{2, \ldots \frac{N}{2}\right\}$ [7]. This paper considers the vertex and edge colouring of a new degree six chordal ring, the optimised degree six
3 -modified chordal ring, $\mathrm{CHR} \mathrm{o}_{3}$. The first section of this paper provides a brief introduction to graph colouring and chordal rings, the second presents the new degree six chordal ring, the third and fourth sections discuss its chromatic numbers and indices respectively, and the last section concludes the paper.

\section{THE OPTIMISED DEGREE SIX 3-MODIFIED CHORDAL RING, $\mathrm{CHR6O}_{3}$}

The degree six 3-modified chordal ring is defined as follows:

Definition 1. The optimised degree six 3-modified chordal ring, $\mathrm{CHRGo}_{3}$, denoted as $\mathrm{CHRGo}_{3}\left(\mathrm{~N}, \mathrm{~s}, h_{1}, h_{2}, h_{3}, h_{4}, h_{5}, h_{6}\right)$ is an undirected circulant graph. The number of nodes, $N$ as well as all chord lengths $h_{1}, h_{2}, h_{3}, h_{4}, h_{5}$, and $h_{6}$ must be divisible by 3 .

Every 3 nodes are grouped into a class.

All the first nodes in the class, $N_{3 i-3}($ e.g. nodes $0,3,6, \ldots)$ are connected together by chords $+h_{1}(\bmod N),-h_{1}(\bmod N),+h_{2}(\bmod N)$, and $-h_{2}(\bmod N)$

All the second nodes in the class, $N_{3 i-2}$ (e.g. nodes $\left.1,4,7, \ldots\right)$ are connected together by chords $+h_{3}(\bmod N),-h_{3}(\bmod N)$, $+h_{4}(\bmod N)$, and $-h_{4}(\bmod N)$.

All the third nodes in the class, $N_{3 i-1}$ (e.g. nodes $\left.2,5,8, \ldots\right)$ are connected together by chords $+h_{5}(\bmod N),-h_{5}(\bmod N)$, $+h_{6}(\bmod N)$, and $-h_{6}(\bmod N)$,

where $i=0,1,2, \ldots$. Further conditions are that $h_{1}<h_{2}, h_{3}<h_{4}, h_{5}<$ $h_{6}$; and $3 \leq h_{1}, h_{2}, h_{3}, h_{4}, h_{5}, h_{6}<\frac{N}{2}$. An example of $\mathrm{CHR} \mathrm{o}_{3}$ is shown in Fig. 1. 


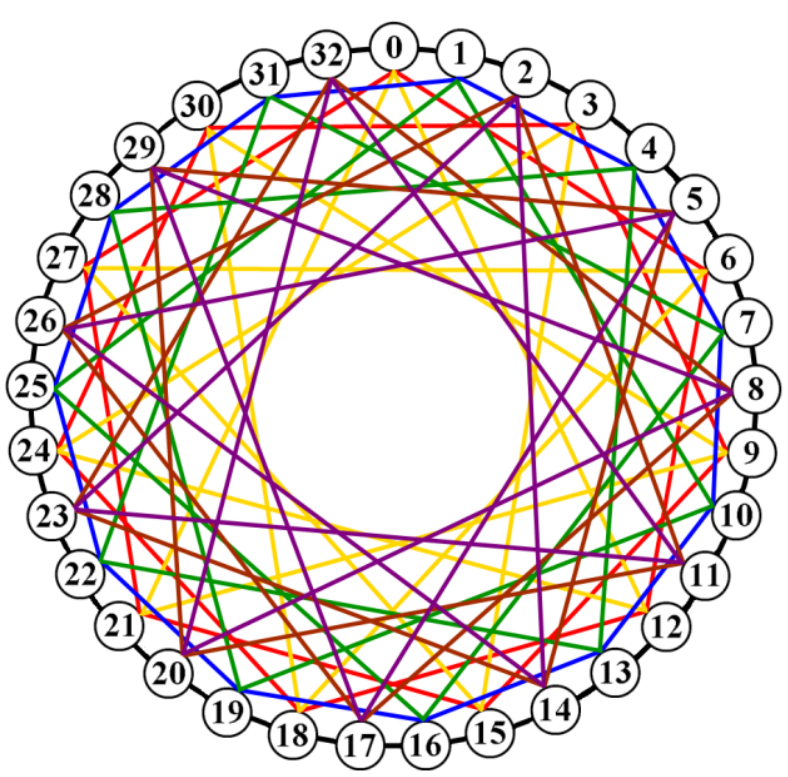

Fig. $1 \mathrm{CHR} o_{3}(33,1,6,12,3,9,9,12)$.

In this graph model, the vertices of a graph represent the processor nodes of the network, and the edges represent the links of the network connecting the processor nodes. Hence, the chromatic number, $\chi\left(\mathrm{CHR6o}_{3}\right)$ and the chromatic index, $\chi^{\prime}\left(\mathrm{CHR6o}_{3}\right)$ in this paper refer to the systematic minimal assigning of colours to the nodes and links of the network model respectively.

\section{THE CHROMATIC NUMBER OF $\mathrm{CHR6o}_{3}, \chi\left(\mathrm{CHR6o}_{3}\right)$}

To investigate the node colouring of optimised degree six 3 -modified chordal ring, $\mathrm{CHR6o}_{3}$, it must first be noted that all the same types of nodes in a class of $\mathrm{CHR}_{3} \mathrm{o}_{3}$ are all connected through the same chords. For example, node $N_{3 i-2}$ can only connect to other nodes, $N_{3 j-2}$ through chords $h_{3}$ and $h_{4}$, where $i, j=0,1,2, \ldots$ and $i \neq j$. Connections to other types of nodes in a class of $\mathrm{CHRGO}_{3}$ are achieved through ring links. Without ring links, the three different nodes in a class of $\mathrm{CHR6o}_{3}$ will form disjoint subgraphs consisting of only one type of node in a class. Furthermore, since all the chords $h_{1}, h_{2}, h_{3}, h_{4}$, $h_{5}$, and $h_{6}$ are divisible by 3 , all three different types of nodes in a class of $\mathrm{CHR} \mathrm{O}_{3}$ has essentially the same types of chord connections.

Node colouring is the systematic process of labelling network nodes such that no two adjacent nodes are assigned the same colour, and the minimum number of colours used is the chromatic number of the network. Despite its symmetrical properties, conditions to achieve a certain $k$-colouring in $\mathrm{CHR} \mathrm{o}_{3}$ cannot be proposed due to its definition. The network size and chord lengths being divisible by 3 , as well as there being 3 different types of nodes in a class of $\mathrm{CHRGo}_{3}$ lead to irregular alternations between labelled nodes. The common multiples also cause the existence of many smaller cycles within large networks of $\mathrm{CHR} \mathrm{o}_{3}$. The symmetrical properties presented earlier however, still aid in developing lower bounds for the chromatic number of $\mathrm{CHR}_{3} \mathrm{O}_{3}$. For example, without ring links, $\mathrm{CHR}_{3} o_{3}(33,1,6,12,3,9,9,12)$ from Fig. 1 will be decomposed into three types of disjoint subgraphs: $\mathrm{H}_{3 i-3}$ consisting of only $\mathrm{N}_{3 i-3}$ nodes, $\mathrm{H}_{3 i-2}$ consisting of only $\mathrm{N}_{3 i-2}$ nodes, and $H_{3 i-1}$ consisting of only $N_{3 i-1}$ nodes. The independent colouring of nodes in these subgraphs is shown in Fig. 2, Fig. 3, and Fig. 4.

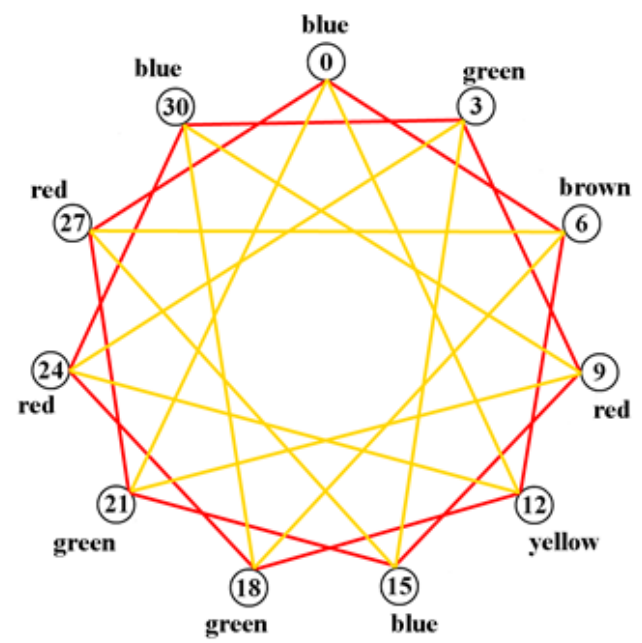

Fig. 2 Subgraph $\mathrm{H}_{3 i-3}$ of $\mathrm{CHR}_{6} o_{3}(33,1,6,12,3,9,9,12)$.

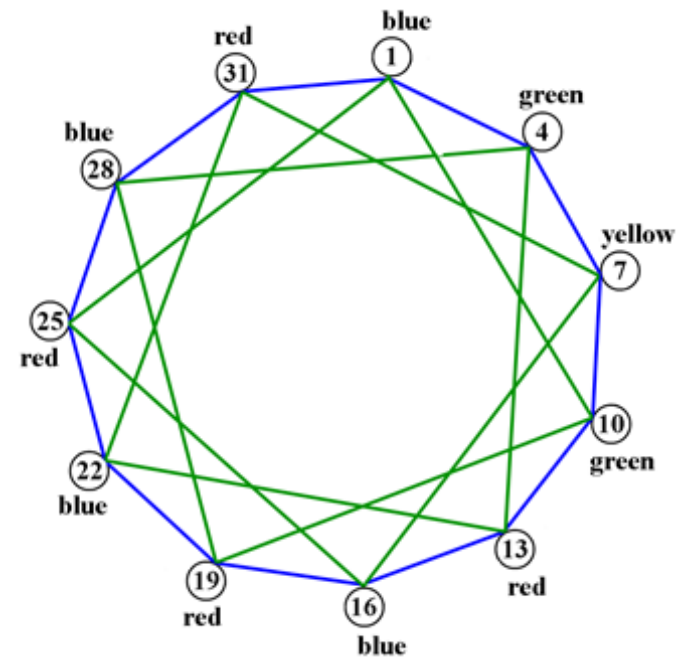

Fig. 3 Subgraph $\mathrm{H}_{3 i-2}$ of $\mathrm{CHR}_{3}(33,1,6,12,3,9,9,12)$.

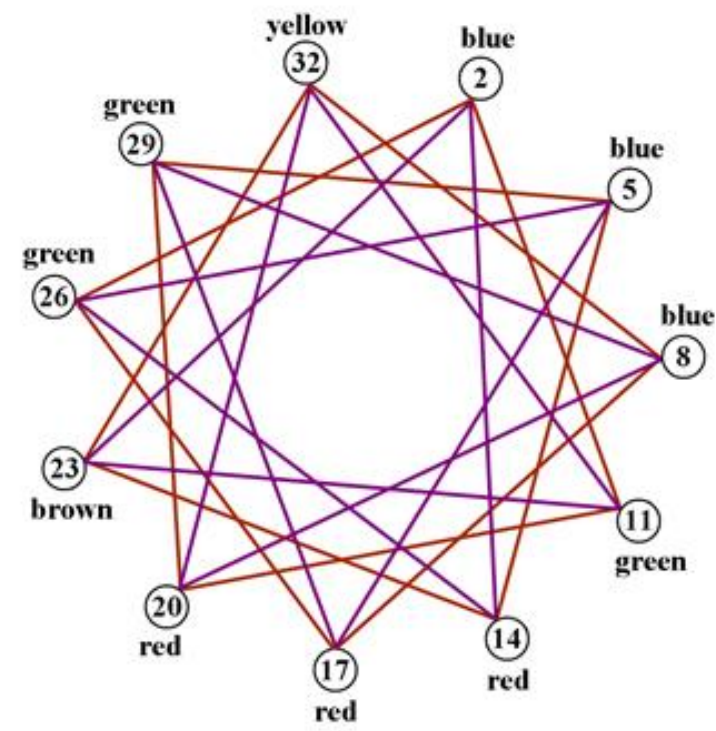

Fig. 4 Subgraph $\mathrm{H}_{3 i-1}$ of $\mathrm{CHR}_{3}(33,1,6,12,3,9,9,12)$.

Based on Fig. 2, Fig. 3, and Fig. 4, the chromatic numbers of the subgraphs of $\mathrm{CHR} \mathrm{o}_{3}(33,1,6,12,3,9,9,12)$ are $\chi\left(H_{3 i-3}\right)=5$, 
$\chi\left(H_{3 i-2}\right)=4$, and $\chi\left(H_{3 i-1}\right)=5$. Expanding on the concept that the chromatic number of a graph $G$ is the upper boundary of that of its subgraph $H$ [8], the lower bound for the chromatic number of $\mathrm{CHR6o}_{3}$ is given by the largest chromatic number of its three subgraphs.

$$
\chi\left(\mathrm{CHR} \mathrm{o}_{3}\right) \geq \max \left\{\chi\left(\mathrm{H}_{3 i-3}\right), \chi\left(\mathrm{H}_{3 i-2}\right), \chi\left(\mathrm{H}_{3 i-1}\right)\right\}
$$

Hence, the lower bound for the chromatic number of $\mathrm{CHR} \mathrm{o}_{3}(33,1,6,12,3,9,9,12)$ is 5 . The highest degree subgraphs composed of chords were used because chords largely determine the connectivity in chordal rings, which in this case only connect the same nodes of a class. This provides a very good estimation for the lower bound of the chromatic number of $\mathrm{CHR6o}_{3}$, which tends to fluctuate depending on network sizes and chord combinations. Brook's Theorem was used to determine an upper bound of $\chi\left(\mathrm{CHRGo}_{3}\right)$. It states that if a graph is connected and undirected, its chromatic number is at most its maximum degree, or its maximum degree plus 1 if the graph contains an odd cycle [8]. $\mathrm{CHR} \mathrm{o}_{3}$ is a degree six chordal ring which has both even and odd networks. By extension, their Hamiltonian Circuits are even cycles and odd cycles respectively. The upper boundaries for chromatic numbers are given by

$$
\chi\left(\mathrm{CHR} \mathrm{o}_{3}\right)_{\text {even }} \leq 6
$$

for even networks of $\mathrm{CHR6o}_{3}$; and

$$
\chi\left(\mathrm{CHR} 6 \mathrm{o}_{3}\right)_{\text {odd }} \leq 7
$$

for odd networks of $\mathrm{CHR}_{3} \mathrm{o}_{3}$. In network planning, a low chromatic number is favourable to a high one, as it aids the minimisation of the completion times of processes. The finish time of a node is the largest colour assigned to it, while the finish time of all the nodes in the network to work on a distributed task is the sum of the colouring [9].

\section{THE CHROMATIC INDEX OF $\mathrm{CHR6o}_{3}, \chi^{\prime}\left(\mathrm{CHR6o}_{3}\right)$}

The minimal number of link colourings for a simple connected graph such that no two links are assigned the same colour is defined as its chromatic index, and is important in reducing bottlenecks by processor scheduling [10].

Theorem 1. The lower bound for the chromatic index of $\mathrm{CHRGo}_{3}$, $\chi^{\prime}\left(\mathrm{CHR6o}_{3}\right)$, is 6 .

Proof:

The optimised degree six 3 -modified chordal ring, $\mathrm{CHR}_{3} \mathrm{o}_{3}$ is a homogenous network where all nodes have six links to other nodes in the network topology. Hence, $\mathrm{CHR} \mathrm{o}_{3}$ is modelled after a 6 -regular graph and each of the six links will have to be assigned a different colour and at least six different colours are required.

Theorem 1 applies to the case of an even network size of $\mathrm{CHR}_{3}$ with only even chord lengths, where its network size, $N$ must be an even multiple of any of its chord lengths. In this case, any one of the chord lengths will generate only cycles with an even number of edges among the same nodes in a class of an even network of $\mathrm{CHR6o}_{3}$, as an even number can be generated by the multiplication of two even numbers. A cycle with an even number of edges is assigned the alternation of two colours. Since there are two cycles at every node in a class of $\mathrm{CHR} \mathrm{o}_{3}$ of two different lengths, and also considering that all the nodes in a class of $\mathrm{CHR}_{\mathrm{O}} \mathrm{o}_{3}$ are disjoint if ring edges are not considered, every node will have four different colours. The ring edges, which make up an even cycle with an even number of links can then be assigned two different colours, leading to the chromatic index, $\chi^{\prime}$ in this case being $4+2=6$. However, this argument does not apply to odd network sizes of $\mathrm{CHR6o}_{3}$ with only odd chord lengths, where its network size, $N$ must be an even multiple of any of its chord lengths, because 3 colours will be required for its ring links instead of 2 .
Theorem 2. For an even network of $\mathrm{CHR} \mathrm{o}_{3}$, its chromatic index, $\chi^{\prime}\left(\mathrm{CHR6o}_{3}\right)$ is 7 .

Proof:

An even network size of $\mathrm{CHR6o}_{3}$ will have a chromatic index of $\chi^{\prime}\left(\mathrm{CHR6o}_{3}\right)=6$ if and only if it exhibits a 1 -factorisation [11]. $\mathrm{CHR}_{\mathrm{O}} \mathrm{o}_{3}$ is modelled after a 6 -regular graph and the lower bound for an even network size, $N$, is 24. Based on the 1 -factorisation conjecture discussed by [12] which states that an $k$-regular graph with an even number of vertices $n$ is contains a 1 -factorisation if

$$
k \geq \frac{n}{2}-1
$$

$\mathrm{CHR} \mathrm{O}_{3}$ has no 1-factorisation because

$$
6<\frac{24}{2}-1
$$

based on its lower bound for even network sizes. The only other option for the chromatic index is $6+1$, consistent with Vizing's Theorem. Fig. 5 shows the links of a degree six 3 -modified chordal ring with an even number of nodes, $\mathrm{CHR}_{3} \mathrm{o}_{3}(30,1,6,12,3,9,6,9)$, labelled with seven colours as an example showing that the six colours, according to the lower bound for the chromatic index of $\mathrm{CHR}_{3} \mathrm{o}_{3}$ are not sufficient.

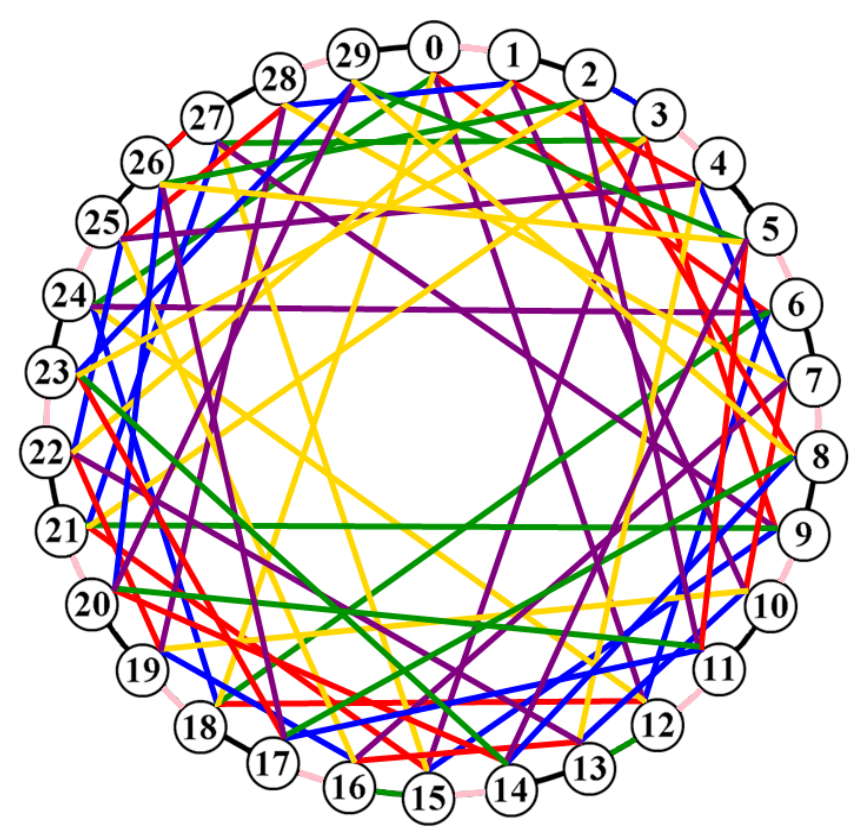

Fig. 5 The Link Colouring of $\mathrm{CHR}_{3} o_{3}(30,1,6,12,3,9,6,9)$.

Theorem 3. For an odd network of $\mathrm{CHR6O}_{3}$, its chromatic index, $\chi^{\prime}\left(\mathrm{CHR6o}_{3}\right)$ is 7 .

Proof:

The proofs discussed by [11] and [13], based on Vizing's Theorem, state that an odd network size of $\mathrm{CHRGo}_{3}$ can be written as $N=2 n+$ 1 for some value of $n=10,13,16, \ldots$. To ensure the graph of odd vertices remains 6 -regular, $6=2 m$ for some number $m$. This means that there are three pairs of edges adjacent to every vertex.

Assume that 6 colours are sufficient for a proper labelling of an odd network of $\mathrm{CHR} \mathrm{oo}_{3}$. By using the degree sum formula, which implies that the number of edges in $C H R \sigma_{3}$ must be divisible by 6 in an odd network,

$$
\frac{6 N}{2}=m(2 n+1)
$$

Rearranging and substituting $N=2 n+1$, 


$$
\begin{aligned}
\frac{m(2 n+1)}{6} & =\frac{N}{2} \\
& =\frac{2 n+1}{2} \\
& =n+\frac{1}{2}
\end{aligned}
$$

Since $n+\frac{1}{2}$ is not a natural number, this implies that there is at least one vertex with the same colour assigned to two if its adjacent vertices. This is a contradiction, which leads to the chromatic index of an odd network of $\mathrm{CHR} \mathrm{o}_{3}$ being $6+1=7$, consistent with Vizing's Theorem. Fig. 6 shows the links of $\mathrm{CHR} \mathrm{o}_{3}(33,1,6,12,3,9,9,12)$, previously shown in Fig. 1, labelled with seven colours, the seventh being black.

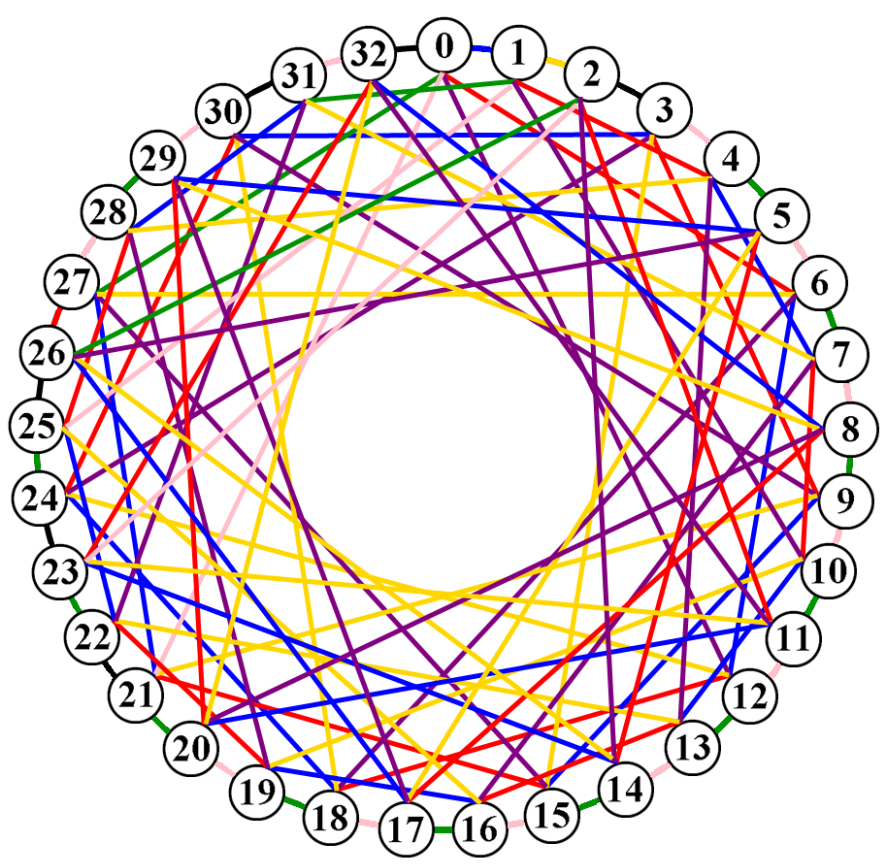

Fig. 6 The Link Colouring of $\mathrm{CHR6o}_{3}(33,1,6,12,3,9,9,12)$

\section{CONCLUSIONS}

A new degree six modified chordal ring was introduced in this paper and its colouring was investigated. The lack of generalisation to determine the exact chromatic number of $\mathrm{CHRGo}_{3}$ was explained and the proposal of bounds for it based on subgraphs and Brook's Theorem were justified. On the other hand, the conditions for $\mathrm{CHR} \mathrm{o}_{3}$ to have a chromatic index of 6 or 7, were discussed and proven. The bounds for chromatic numbers and chromatic indices provide some measure of the processing times and likelihood of bottlenecks in a network of $\mathrm{CHR6o}_{3}$. However, these two measures are purely theoretical and may differ in case studies where many more factors such network sizes come to play. Furthermore, since the exact chromatic number of a particular network of $\mathrm{CHR} \mathrm{o}_{3}$ depends on its network size and chord combinations, suggested further work would be the development of an efficient algorithm to determine its chromatic number for any particular network.

\section{REFERENCES}

[1] Rosen, K. H. (2012). Discrete Mathematics and its Applications. ( $7^{\text {th }}$ Edition). New York: McGraw-Hill.

[2] Van Steen, M. (2010). Graph Theory and Complex Networks: An Introduction. ( $1^{\text {st }}$ Edition). Amsterdam: Maarten van Steen.

[3] R. N. F. Azura, M. Othman, Y. H. Peng, and M. H. Selamat. Malaysian Journal of Mathematical Sciences 4(2) (2010) 147-157.

[4] R. N. Farah, M. Othman, M. H. Selamat. Journal of Computer Science, 6(3) (2010) 279-284.
[5] S. Bujnowski, B. Dubalski, A. Zabludowski, D. Ledziński, T. Marciniak, and J.M. Pedersen. Image Processing and Communications Challenges 2, Advances in Intelligent Systems and Computing, 84 (2010) 435-445.

[6] B. W. Arden and H. Lee. IEEE Trans. Computer, C-30(4) (1981) 291-295.

[7] D. Ledziński, S. Bujnowski, T. Marciniak, J.M. Pedersen, J.G. Lopez. Image Processing and Communications Challenges 5, Advances in Intelligent Systems and Computing 233 (2014) 281-299.

[8] Brooks, R.L. On colouring the nodes of a network. Proceedings of the Cambridge Philosophical Society, Mathematical and Physical Sciences. 1941, 37(2):194-197, Available from, DOI: 10.1017/S030500410002168X.

[9] D. Marx. Periodica Polytechnica, Electrical Engineering 48(1) (2004) 1116.

[10] P. Gupta. International Journal Of Core Engineering \& Management (IJCEM). 1(2) (2014) 27-32.

[11] Jungnickel, D. (2013). Graphs, Networks and Algorithms. (4 ${ }^{\text {th }}$ Edition). Springer.

[12] Chetwynd A. G., Hilton A. J. W. Regular Graphs of High Degree are 1Factorizable. Proceedings of the London Mathematical Society. 1985. 2: 193-206. Available from, DOI: 10.1112/plms/s3-50.2.193

[13] R. Green. (2015). Vizing's Theorem And Edge-Chromatic Graph Theory. 1-8. Obtained from http://math.uchicago.edu/ may/REU2015/REUPapers/Green.pdf 